
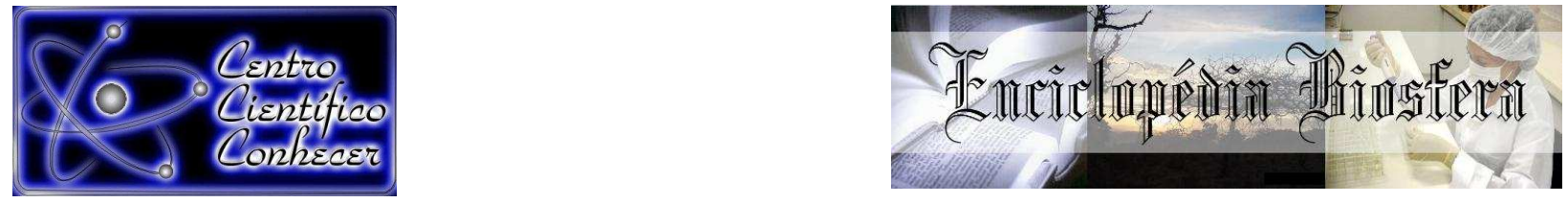

\title{
CARACTERÍSTICAS ESTRUTURAIS DE FOLHAS EM DIFERENTES ESTÁDIOS FENOLÓGICOS DE Tectona grandis L.f.
}

\author{
Daniela Pereira Dias ${ }^{1}$, Karita Kristina Sousa Freitas ${ }^{2}$, Danival Vieira de Freitas ${ }^{3}$ \\ ${ }^{1}$ Professora Doutora do Curso de Engenharia Florestal da Universidade Federal de \\ Goiás - Regional Jataí (danieladias@ufg.br) Jataí - Brasil \\ ${ }^{2}$ Acadêmica do curso de Engenharia Florestal da Universidade Federal de Goiás - \\ Regional Jataí - Brasil \\ ${ }^{3}$ Professor Doutor do Curso de Engenharia Florestal da Universidade Federal de \\ Goiás - Regional Jataí - Brasil
}

Recebido em: 08/04/2016 - Aprovado em: 30/05/2016 - Publicado em: 20/06/2016 DOI: 10.18677/Enciclopedia_Biosfera_2016_022

\begin{abstract}
RESUMO
Mudanças estruturais em folhas de diferentes estádios fenológicos em espécies florestais podem implicar na dinâmica de crescimento e em seu estabelecimento em determinada região. O objetivo deste estudo foi determinar as características estruturais de folhas em diferentes estádios fenológicos de Tectona grandis L.f. (Lamiaceae) plantadas em Jataí - GO. As fontes de nutrientes adicionadas à cova no plantio foram orgânica, química, orgânica+química e controle. Após um ano do plantio de mudas de $T$. grandis, foram determinados atributos biométricos (altura e diâmetro) e características estruturais em folhas em expansão, maduras e senescentes. Os dados foram coletados em janeiro de 2011, durante o período chuvoso. Não houve efeito da adição das fontes nutricionais ao solo sobre os atributos foliares, nem sobre os parâmetros biométricos analisados. $O$ diâmetro e a altura das plantas foram, em média, de 2,66 cm e 1,34 $\mathrm{m}$, respectivamente. Houve $100 \%$ de sobrevivência das mudas de $T$. grandis. A espessura foliar, a massa foliar específica e o índice de esclerofilia aumentaram com o avanço do estádio fenológico das folhas de $T$. grandis. Por outro lado, a área foliar específica e o conteúdo de água foram maiores nas folhas em expansão do que nas maduras e senescentes. $O$ conteúdo de clorofila das folhas foi maior nas folhas maduras, seguidas das senescentes e em expansão. Após um ano do plantio, as árvores jovens de $T$. grandis apresentaram bom estabelecimento inicial em Jataí. Folhas em expansão, maduras e senescentes possuem características estruturais distintas, estratégia essencial para sua sobrevivência e produtividade.
\end{abstract}

PALAVRAS-CHAVE: área foliar específica, conteúdo de água da folha, ecossilvicultura. 


\title{
LEAF STRUCTURAL TRAITS AT DIFFERENT PHENOLOGICAL PHASES OF Tectona grandis L.f.
}

\begin{abstract}
Leaf structural changes at different phenological phases of forest species may involve the growth dynamics and their establishment in a particular region. The aim of this study was to determine the leaf structural traits at different phenological phases of Tectona grandis L.f. (Lamiaceae) planted in Jataí - GO. Four treatments concerning fertilizations with organic, chemistry, organic/chemical and control. At one year after planting were determined biometric attributes (height and diameter) and structural traits in expanding leaves, mature and senescent. Data were collected in January 2011, during the rainy season. There was no effect of adding nutritional sources on the leaf traits and biometric parameters analyzed. Stem diameter and plant height was $2.66 \mathrm{~cm}$ and $1.34 \mathrm{~m}$, respectively. There was $100 \%$ survival of $T$. grandis seedlings. Leaf thickness, mass per area and sclerophylly index increased with the phenological stage of $T$. grandis leaves. Moreover, specific leaf area and the water content was greater in the expanding leaves than in mature and senescent leaves. Leaf chlorophyll content was higher in mature leaves, followed by senescent and expanding. After one year of planting $T$. grandis showed potential of establishment in Jataí. Expanding leaves, mature and senescent have distinct structural traits, strategies to improve survival and productivity.
\end{abstract}

KEYWORDS: ecosilviculture, leaf water content, specific leaf area.

\section{INTRODUÇÃO}

Os plantios de Tectona grandis L.f. (Lamiaceae), conhecida comumente como teca, iniciaram-se no Brasil no final da década de 1960, na região do município de Cáceres - MT, onde as condições climáticas são semelhantes às da Índia, local de origem da espécie. Além das condições climáticas favoráveis, o solo de melhor fertilidade e os tratos silviculturais mais adequados e intensos contribuíram para reduzir o ciclo de produção de 80 anos, na região de origem da teca, para apenas 25 anos, na região de Cáceres-MT (ANGELI \& STAPE, 2003, BEZERRA et al., 2011). Apesar disso, segundo a Indústria Brasileira de Árvores (IBÁ 2015), em 2014, havia 87.499 ha plantados com a cultura de teca no Brasil. Praticamente apenas as regiões Norte e Centro-Oeste do país possuem mais de 67.000 ha plantados com esta cultura, com incremento médio anual de $15 \mathrm{~m}^{3} /$ ha.ano em 2012 (ABRAF, 2013). Apesar do Centro-oeste brasileiro possuir $67,4 \%$ da área plantada de teca, segundo o anuário da ABRAF, os plantios de teca no estado de Goiás ainda são incipientes. Dessa forma, estudos que investigam fatores que influenciam o desenvolvimento e a produtividade de $T$. grandis em Goiás se tornam importantes, sobretudo, devido à existência de poucas áreas destinadas a esta cultura no estado.

T. grandis é uma espécie arbórea da floresta tropical com hábito heliófilo e decíduo, com queda das folhas no período de menor precipitação pluviométrica (CARVALHO, 2006). As plantas decíduas são definidas como aquelas com folhas de vida curta, associadas a plantas que produzem folhas de forma contínua à medida que crescem (SOUZA et al., 2015). Quanto menor o tempo de vida foliar de uma espécie, maior a taxa de crescimento quando a planta é jovem. Comparando-se plantas que possuem folhas decíduas com as que possuem folhas perenifólias, as decíduas tendem a investirem menos em materiais que protejam e contribuam para 
seu tempo de vida, como lignina e fibras, e investem mais em materiais que contribuam para taxa fotossintética e de crescimentos altos (enzimas fotossintetizantes e outras proteínas) (SOUZA et al., 2015, VERGUTZ et al., 2012). Destaca-se também que plantas decíduas habitam geralmente locais com solos ricos em nutrientes e com boa disponibilidade de água.

Os estudos de fenologia foliar descrevem propriedades importantes, como o grau de deciduidade, o período de emergência, expansão, e mortalidade e longevidade foliar (KIKUZAWA, 1995, ROSSATO, 2013). Variações na fenologia foliar podem ser consideradas estratégicas para maximizar a sobrevivência e a produtividade (ROSSATO \& KOLB, 2009), uma vez que o balanço de carbono e o crescimento da planta estão intimamente relacionados com os padrões de surgimento, abscisão e longevidade foliar (REICH, 1995). Assim, estudos sobre a fenologia foliar são essenciais para compreensão dos padrões de crescimento da árvore, do transporte de água, e da produtividade primária líquida $(\mathrm{REICH}, 1995$, ROSSATO \& FRANCO, 2008, CHATURVEDI et al., 2011).

Avaliações do potencial de estabelecimento, da dinâmica de crescimento e dos principais eventos fenológicos característicos de uma espécie florestal fornecem subsídios científicos para uma análise de seu potencial de adaptação em uma determinada região e definição das futuras práticas silviculturais a serem recomendadas (MACEDO et al., 2007). Dessa forma, objetivou-se determinar as características estruturais de folhas de diferentes estádios fenológicos de T. grandis um ano após o plantio em Jataí - GO.

\section{MATERIAL E MÉTODOS}

O estudo foi conduzido em um plantio de Tectona grandis L.f. (Lamiaceae) no

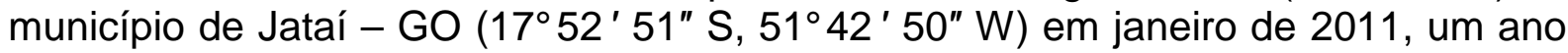
após o plantio das mudas. Os solos onde foram plantadas as mudas de $T$. grandis foram caracterizados como latossolo vermelho escuro distroférrico, com $\mathrm{pH}=5,6$, teor de matéria orgânica no solo de $31,01 \mathrm{~g} / \mathrm{kg}$, soma de bases de $8,96 \mathrm{Cmolc} / \mathrm{dm}^{3}$, capacidade de troca de cátions de $15,64 \mathrm{mg} / \mathrm{dm}^{3}$ e saturação de bases de $57,28 \%$. Segundo a classificação de Köepen, o clima é do tipo Cw, mesotérmico, com estação seca e chuvosa definidas. No município de Jataí, historicamente, a pluviosidade mensal varia de 11,5 a $319 \mathrm{~mm}$, com temperatura média de 22,2 ${ }^{\circ} \mathrm{C}$.

$\mathrm{Na}$ ocasião do plantio, em janeiro de 2010, as mudas foram plantadas sob três diferentes fontes de nutrientes (orgânica; química; química e orgânica) e um tratamento controle sem adição de fonte nutricional. A fonte química de nutrientes consistiu em $300 \mathrm{~g}$ por cova de superfosfato simples. A fonte orgânica foi de $3,00 \mathrm{~kg}$ de esterco bovino curtido por cova, a fonte química/orgânica continha $150 \mathrm{~g}$ de superfosfato simples e $1,50 \mathrm{~kg}$ de esterco bovino.

Foram determinados atributos biométricos das árvores (diâmetro e altura) e morfofisiológicos das folhas (índice de clorofila total, área foliar específica, conteúdo de água, índice de esclerofilia, espessura foliar e massa foliar específica) em folhas de T. grandis em diferentes estádios fenológicos (em expansão, madura e senescente). $O$ diâmetro das árvores foi mensurado com o auxílio de paquímetro digital a $30 \mathrm{~cm}$ de altura do solo e a altura medida com uma régua milimetrada.

$\mathrm{O}$ índice de clorofila total foi determinado com o uso de um medidor eletrônico portátil do teor de clorofila (Clorofilog, CFL1030 - Falker, Brasil). Foram realizadas 
duas medições do índice de clorofila total por folha em cada estádio fenológico (folha em expansão, madura e senescente). A espessura das folhas de diferentes estádios fenológicos foi obtida com um micrômetro digital após a coleta. As folhas foram coletadas e pesadas (massa verde - Mv) e, posteriormente, foram levadas ao Laboratório de Ecologia e Ecofisiologia Florestal para secagem, em estufa de circulação forçada de ar à $65^{\circ} \mathrm{C}$. Após atingirem massa constante, as amostras foram novamente pesadas (massa seca - Ms). A área foliar específica (AFE) foi calculada por meio da razão entre área foliar $\left(\mathrm{cm}^{2}\right)$ e massa de matéria seca $(\mathrm{g})$, para as folhas em expansão, maduras e senescentes. $O$ cálculo do conteúdo de água (CA) na folha foi obtido conforme a fórmula: CA $(\%)=100^{*}(\mathrm{Mv}-\mathrm{Ms}) / \mathrm{Mv}$. A massa foliar específica foi calculada pela razão entre a massa foliar seca e a área foliar.

O delineamento experimental utilizado foi o inteiramente casualizado (DIC), com quatro tratamentos e quatro repetições. Os dados foram submetidos à análise de variância (ANOVA) e, posteriormente, foi aplicado o Teste de Tukey. A parcela foi composta por nove árvores. Para as características foliares foram coletadas seis folhas por indivíduo estudado, duas em cada estádio fenológico.

\section{RESULTADOS E DISCUSSÃO}

Não houve influência da fonte nutricional adicionada ao solo no plantio das mudas de $T$. grandis sobre os atributos biométricos e estruturais das folhas em diferentes estádios fenológicos analisados $(p>0,05)$. Isto pode sugerir que, após um ano do plantio das mudas, a adição de fontes nutricionais não influenciou o crescimento em diâmetro e altura, nem as características foliares de teca.

Como se tratou de uma avaliação pontual, durante o estabelecimento das mudas, os parâmetros observados neste estudo podem apresentar variação ao longo do tempo com as diferentes fontes nutricionais. RIBEIRO et al. (2006) não encontraram diferença na sobrevivência de mudas de teca, sete meses após o plantio, submetidas a diferentes tratamentos de adubação. PINTO et al. (2001) relataram que a adubação com $300 \mathrm{~g} /$ cova de superfosfato simples propiciou um crescimento superior em $12 \%$ em relação a outros tipos de fertilização em um povoamento de teca instalado em Carlópolis - PR.

As árvores jovens de T. grandis, após 12 meses do plantio, apresentaram $100 \%$ de sobrevivência, altura média de $1,34 \mathrm{~m}$ e diâmetro médio de $2,66 \mathrm{~cm}$. No estudo conduzido por MACEDO et al. (2005), onde foram testados diferentes espaçamentos de plantio de teca, a sobrevivência média foi de $65,9 \%$, o diâmetro variou de 2,89 a $3,50 \mathrm{~cm}$ e a altura variou de 2,1 a 2,8 m, ambos medidos após 24 meses do plantio. Em outro estudo de MACEDO et al. (2007), após 12 meses do plantio, teca apresentou altura de $1,25 \mathrm{~m}$ e diâmetro, medido a $20 \mathrm{~cm}$ do solo, de $4,12 \mathrm{~cm}$. Tais dados demonstram o bom estabelecimento inicial da espécie em Jataí $-\mathrm{GO}$.

Os dados de diâmetro e altura das árvores são utilizados para a estimativa do volume total, um dos parâmetros utilizados para comparação quantitativa e qualitativa do desenvolvimento de teca plantada em diferentes áreas do mundo (PELISSARI et al., 2013). Além disso, o diâmetro e a altura das árvores também podem auxiliar na estimativa e quantificação do cerne das árvores de teca, responsável pela importância econômica da espécie, o que é determinante para o 
rendimento de madeira serrada (LEITE et al., 2011). SANQUETA et al. (2015) indicam a inclusão de uma segunda medição de diâmetro para uma melhor estimativa de volume, mesmo sem a inclusão da variável altura, em plantios jovens de teca.

As folhas de estádios fenológicos diferentes de $T$. grandis apresentaram índice de clorofila total (ICT) distintos (Figura 1). Os maiores valores de ICT foram encontrados nas folhas maduras, seguidas das senescentes e das em expansão. Vale ressaltar que as folhas em expansão de $T$. grandis apresentam coloração arroxeada. O teor de clorofila, assim como a espessura e idade da folha, influencia a capacidade fotossintética da planta, o que confere às folhas em fase inicial de crescimento ou em fase de senescência taxas menores de fotossíntese em relação à folhas maduras completamente expandidas (MARENCO et al., 2014). Durante o desenvolvimento da folha, ocorre aumento no teor de clorofila, porém em folhas senescentes observou-se a degradação da clorofila.
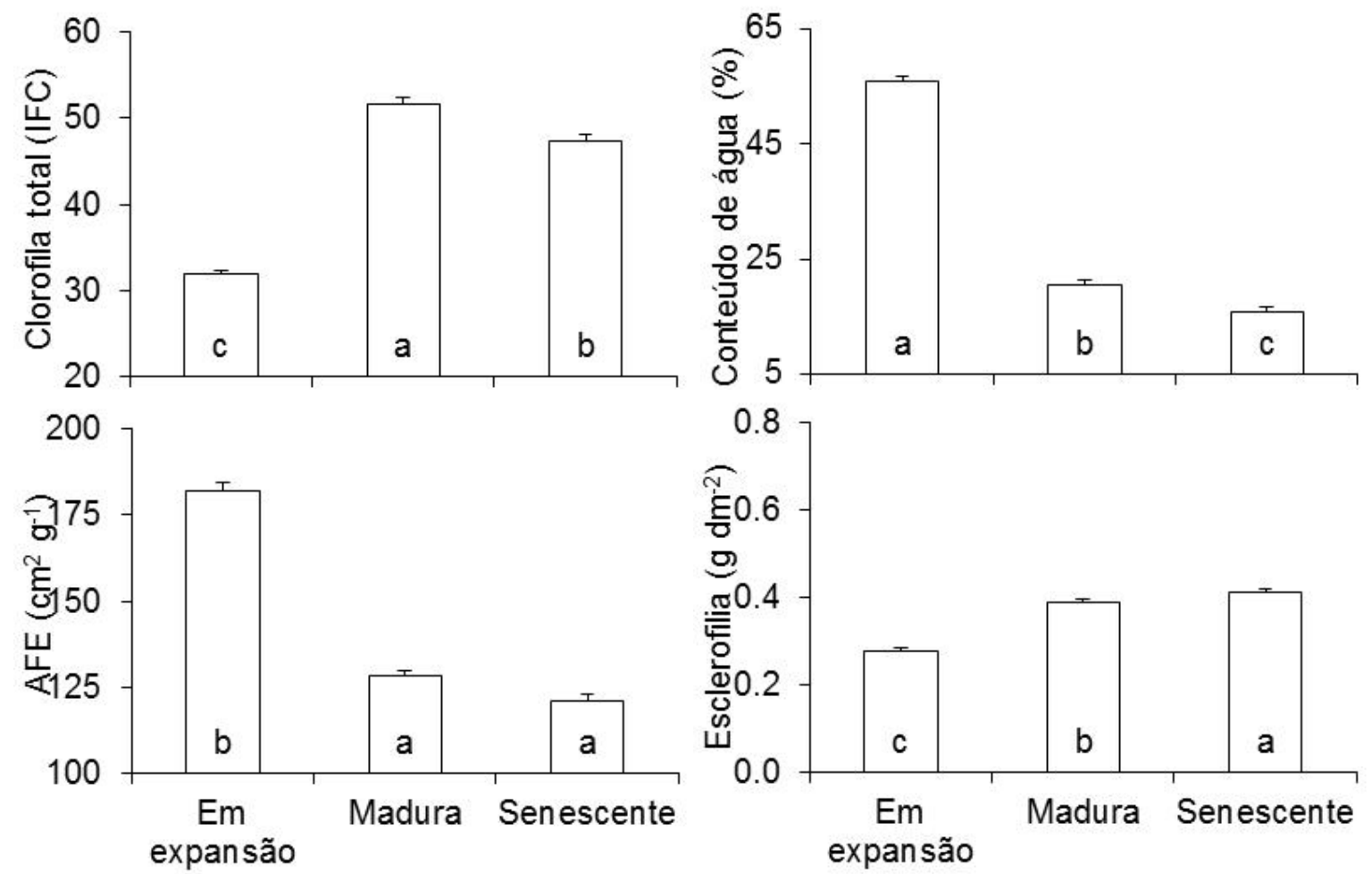

FIGURA 1 - Índice de clorofila total (ICF), área foliar específica (AFE, $\left.\mathrm{cm}^{2} \cdot \mathrm{g}^{-1}\right)$, conteúdo de água (\%) e índice de esclerofilia ( $\left.\mathrm{g} \cdot \mathrm{cm}^{-2}\right)$ em folhas em expansão, maduras e senescentes de árvores jovens de Tectona grandis L.f. plantadas em Jataí - GO. Médias seguidas de mesma letra não diferem entre si pelo teste de Tukey a $5 \%$ de probabilidade. Desvios acima das barras representam o erro padrão da média.

Assim como o índice de clorofila total, o conteúdo de água (CA) das folhas variou em função do estádio fenológico (Figura 1). Neste estudo, quanto mais jovem o tecido foliar maior a quantidade de água encontrada. Comparadas ao CA das folhas maduras, o CA das folhas em expansão aumentou $173,21 \%$ enquanto que 0 $\mathrm{CA}$ das folhas senescentes reduziu $22,23 \%$. Isto ocorre porque o amadurecimento das folhas provoca aumento de tecidos estruturais (lignina, fibras, celulose), o que 
garante maior dureza à folha. Durante a senescência foliar ocorre a degradação gradual das proteínas foliares, favorecendo as partes mais jovens da planta. Por exemplo, o nitrogênio migra das folhas mais velhas para as que as folhas em expansão, mantendo assim o nitrogênio contido nas proteínas ligadas à clorofila. $\mathrm{A}$ cor verde desaparece com o envelhecimento dos cloroplastos das folhas porque com a decomposição do complexo proteína-pigmento, as folhas ficam amareladas, sendo os produtos da quebra da clorofila translocadas para o vacúolo (LARCHER, 1980).

A área foliar específica (AFE) das folhas maduras e senescentes de $T$. grandis não diferiu entre si, entretanto a AFE das folhas em expansão foram superiores nos outros estádios fenológicos (Figura 1). Valores de AFE de folhas de $T$. grandis na Índia bem inferiores foram encontrados por CHATUVERDI et al. (2013), em média $65 \mathrm{~cm}^{2} \mathrm{~g}^{-1}$. Para HENDRIK \& JONG (1999), folhas com menores valores de AFE apresentam paredes celulares mais espessas, maior concentração de metabólitos secundários, folhas menos espessas, o que, consequentemente, confere às folhas tecidos mais estruturados e maior dureza.

Quanto mais avançado o estádio fenológico das folhas maior foi o índice de esclerofilia (IE) de T. grandis (Figura 1). Segundo RIBEIRO et al. (2012), a maior esclerofilia das folhas pode estar relacionada a maior espessura dos parênquimas paliçádico e lacunoso, assim como maior eficiência para reduzir a transpiração e aproveitamento da radiação incidente. Independente do estádio fenológico foliar da espécie estudada, os valores de IE das folhas de diferentes estádios fenológicos permitem classificá-las como mesófilas, o que confere característica adequada para regiões de boa disponibilidade de água.

A espessura foliar fresca de $T$. grandis variou entre os estádios fenológicos foliares (Figura 2). O aumento da espessura foliar está diretamente associado ao desenvolvimento foliar, sobretudo ao acúmulo de carbono. Além disso, em espécies decíduas, a espessura foliar, bem como a área foliar específica e a densidade da madeira, têm sido consideradas características importantes para garantir a adaptação das plantas à seca (SILVA et al., 2014).
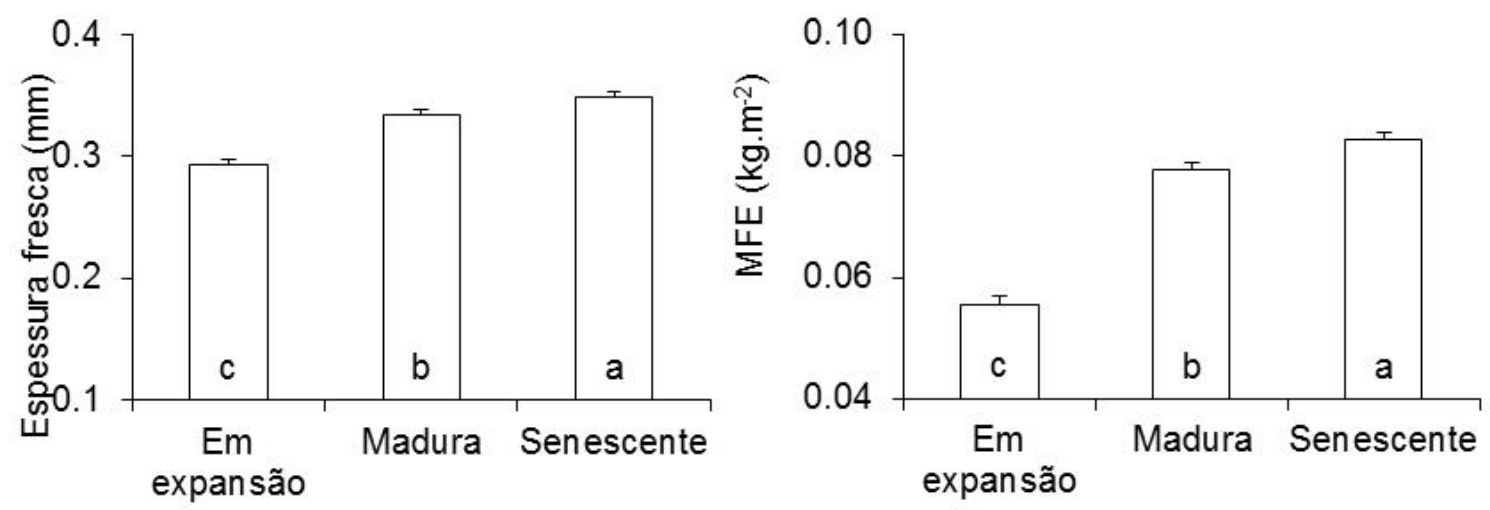

FIGURA 2 - Espessura fresca (mm) e massa foliar específica (MFE, kg.m ${ }^{-2}$ ) das folhas em expansão, maduras e senescentes de Tectona grandis L.f. plantadas em Jataí - GO. Médias seguidas de mesma letra não diferem entre si pelo teste de Tukey a $5 \%$ de probabilidade. Desvios acima das barras representam o erro padrão da média. 
Por outro lado, quanto mais avançado o estádio fenológico das folhas maior foi a sua massa foliar específica (MFE) (Figura 2). As folhas em expansão possuem maior conteúdo de água e menor matéria seca por unidade de área, quando comparadas às folhas senescentes. Além disso, folhas de espécies decíduas, como a teca, apresentam menor MFE quando comparadas às sempre verdes, o que pode funcionar como uma estratégia de evite e tolerância à seca (SOUZA et al., 2015, FU et al., 2012).

O lançamento de folhas novas durante o período chuvoso concomitante ao processo de senescência geneticamente programada de folhas seguindo a ordem cronológica de desenvolvimento, após um ano do plantio das mudas de teca no campo, confirma a variação das características estruturais em folhas de estádios fenológicos diferentes em uma mesma árvore em sua fase juvenil. Outros trabalhos se fazem necessários visando investigar a longevidade das folhas e seu efeito sobre a assimilação de carbono, determinar a concentração e redistribuição de nutrientes minerais nos estádios fenológicos analisados, entre outros. Assim, práticas silviculturais que influenciam positivamente a produtividade da espécie poderão ser recomendadas.

\section{CONCLUSÕES}

As diferentes fontes nutricionais utilizadas no plantio das mudas de Tectona grandis não influenciam, após um ano do plantio, o crescimento e as características estruturais foliares.

A sobrevivência de $100 \%$ das mudas de T. grandis plantadas em Jataí, bem como os valores de a altura e diâmetro das árvores após um ano do plantio, indicam seu bom estabelecimento inicial em Jataí - GO.

\section{REFERÊNCIAS}

ANGELI A.; STAPE, J. L. Tectona grandis (Teca). In: Identificação de espécies florestais. Piracicaba: IPEF, $2003 . \quad$ Disponível em: http://www.ipef.br/identificacao/tectona.grandis.asp. Acesso em: 04 mar. 2016.

ASSOCIAÇÃO BRASILEIRA DE PRODUTORES DE FLORESTAS PLANTADAS (ABRAF). Anuário estatístico da ABRAF 2013, ano base 2012. Brasília: ABRAF, 2013.

BEZERRA, A. F.; MILAGRES, F. R.; SILVA, M. L.; LEITE, H. G. Análise da viabilidade econômica de povoamentos de Tectona grandis submetidos a desbastes no Mato Grosso. Cerne, v. 17, n. 4, p. 583-592, 2011. Disponível em: <https://dx.doi.org/10.1590/S0104-77602011000400018>. doi: 10.1590/S010477602011000400018

CARVALHO, M. S. Manual de Reflorestamento. Belém: SEBRAE, 2006. 140 p.

CHATURVEDI, R. K.; RAGHUBANSHI, A. S.; SINGH, J. S. Leaf attributes and tree growth in a tropical dry forest. Journal of Vegetation Science, v. 22, n. 5, p. 917931, 2011. Disponível em: <http://dx.doi.org/10.1111/j.1654-1103.2011.01299.x>. doi: 10.1111/j.1654-1103.2011.01299.x 
CHATURVEDI, R. K.; PRASAD, S.; RANA, S.; OBAIDULLAH, S. M.; PANDEY, V.; $\mathrm{SINGH}, \mathrm{H}$. Effect of dust load on the leaf attributes of the tree species growing along the roadside. Environmental monitoring and assessment, v. 185, n. 1, p. 383-391, 2013. Disponível em: < http://link.springer.com/article/10.1007/s10661-012-2560-x>. doi: $10.1007 / \mathrm{s} 10661-012-2560-x$

DE LA RIVA E. G.; OLMO M.; POORTER H.; UBERA J. L.; VILLAR R. Leaf mass per area (LMA) and its relationship with leaf structure and anatomy in 34 Mediterranean woody species along a water availability gradient. PLoS ONE, v. 11, n. 2, p. 1-18, 2016. Disponível em: <http://dx.doi.org/10.1371/journal.pone.0148788>. doi: 10.1371/journal.pone.0148788

FU, P.L.; JIANG, Y. J.; WANG, A. Y.; BRODRIBB, T. J.; ZHANG, J. L.; ZHU, S. D.; $\mathrm{CAO}, \mathrm{K}$. F. Stem hydraulic traits and leaf water-stress tolerance are co-ordinated with the leaf phenology of angiosperm trees in an Asian tropical dry karst forest. Annals of Botany, v. 110, n. 1, p. 1-11, 2012. Disponível em: < https://aob.oxfordjournals.org/content/early/2012/05/13/aob.mcs092.full.pdf+html>. doi: 10.1093/aob/mcs092

HENDRIK, P.; JONG, R. A comparison of specific leaf area, chemical composition and leaf construction costs of field plants from 15 habitats differing in productivity. New Phytologist, v. 143, n. 1, p. 163-176, 1999.

\section{INDÚSTRIA BRASILEIRA DE ÁRVORES (IBÁ). Relatório da Indústria Brasileira de Árvores. São Paulo; 2015. Disponível em: http://iba.org/images/shared/iba_2015.pdf. Acesso em: 05 abr. 2016.}

KIKUZAWA, K. Leaf phenology as an optimal strategy for carbon gain in plants. Canadian Journal of Botany, v. 73, p. 158-163, 1995. Disponível em: < http://www.nrcresearchpress.com/doi/abs/10.1139/b95-019\#.V0x60SEerVQ>. doi: 10.1139/b95-019

LARCHER, W. Ecofisiologia vegetal. São Carlos: RiMa, 2006. 531 p.

LEITE, H. G.; OLIVEIRA-NETO, R. R.; MONTE, M. A.; FADIN, L.; ALCANTARA, A. M.; BINOTI, m. L. M. S.; CASTRO, R. V. O. Modelo de afilamento de cerne de Tectona grandis L.f. Scientia Forestalis, v. 39, n. 89, p. 53-59, 2011. Disponível em:<http://www.bibliotecaflorestal.ufv.br/bitstream/handle/123456789/16177/Scientia _Forestalis_v39_n89_p53-59_2011.pdf? sequence=1\&isAllowed=y>.

MACEDO, R. L. G.; GOMES, J. E.; VENTURIN, N.; SALGADO, B. G. Desenvolvimento inicial de Tectona grandis L.f. (teca) em diferentes espaçamentos no município de Paracatu, MG. Cerne, v. 11, n. 1, p. 61-69, 2005.

MACEDO, L. R. G.; VENTURIN, N.; GOMES, J. E.; SALGADO, B. G.; KRUSCHEWSKY, G. C. Phenology and initial growth of Tectona grandis L. F (teca) in Lavras - MG. Cerne, v. 13, n. 5, p. 81-90, 2007. 
MARENCO, R. A.; ANTEZANA-VERA, S. A.; GOUVÊA, P. R. dos S.; CAMARGO, M. A. B.; OLIVEIRA, M. F. de; SANTOS, J. K. da S. Fisiologia de espécies florestais da Amazônia: fotossíntese, respiração e relações hídricas. Revista Ceres, v. 61, n. 7, p. 786-799, 2014. Disponível em: <https://dx.doi.org/10.1590/0034737×201461000004>. doi: 10.1590/0034-737×201461000004.

PELISSARI, A. L.; CALDEIRA, S. F.; DRESCHER, R. Desenvolvimento quantitativo e qualitativo de Tectona Grandis L.f. em Mato Grosso. Floresta e Ambiente, v. 20, n. 3, p. 371-383, 2013. Disponível em: <http://dx.doi.org/10.4322/floram.2013.027>. doi: $10.4322 /$ floram.2013.027

PINTO, A. F.; RODIGHERI, H. R. Desenvolvimento da teca (Tectona grandis) e mogno (Swietenia macrophylla) consorciados, em diferentes níveis de adubação fosfatada, no município de Carlópolis, PR. Embrapa Florestas. Comunicado Técnico, 2001.

REICH, P. B.; WALTERS, M. B.; KL, B. D.; ELLSWORTH, D. S. Different photosynthesis-nitrogen relations in deciduous hardwood and evergreen coniferous tree species. Oecologia, v. 104, n. 1, p. 24-30, 1995. Disponível em: < http://link.springer.com/article/10.1007/BF00365558>. doi: 10.1007/BF00365558

RIBEIRO, F. A.; MACEDO, R. L. G.; VENTURIM, N.; MORAIS, V. M.; GOMES, J. E; YOSHITAMI JR, M. Efeitos da adubação de plantio sobre o estabelecimento de mudas de Tectona grandis L. f. (teca). Revista Científica Eletrônica de Engenharia Florestal, v. 4, n. 7, p. 1-13, 2006.

RIBEIRO, M. de N. O.; CARVALHO, S. P. de; PEREIRA, F. J.; CASTRO, E. M. de. Anatomia foliar de mandioca em função do potencial para tolerância à diferentes condições ambientais. Revista Ciência Agronômica, v. 43, n. 2, p. 354-361, 2012. Disponível em: <http://dx.doi.org/10.1590/S1806-66902012000200019>. doi: $10.1590 /$ S1806-66902012000200019

ROSSATTO, D. R. Seasonal patterns of leaf production in co-occurring trees with contrasting leaf phenology: time and quantitative divergences. Plant Species Biology, v. 28, n.2, p. 138-145, 2013. Disponível em: 10.1111/j.14421984.2012.00373.x. Doi: 10.1111/j.1442-1984.2012.00373.x.

ROSSATTO, D. R.; ROSANA M. K. An evergreen neotropical savanna tree (Gochnatia polymorpha, Asteraceae) produces different dry-and wet-season leaf types. Australian Journal of Botany, v. 57, n. 5, p. 439-443, 2009. Disponível em: http://dx.doi.org/10.1071/BT09045. doi: 10.1071/BT09045

ROSSATTO, D. R.; FRANCO, A. C. Expansão e mortalidade de ramos em espécies arbóreas do cerrado sensu stricto. Brazilian Journal of Botany, v. 31, n. 4, p. 715719, 2008. Disponível em: <https://dx.doi.org/10.1590/S0100-84042008000400016>. Doi: $10.1590 / \mathrm{S} 0100-84042008000400016$ 
SANQUETTA, M. N. I.; SANQUETTA, C. R.; MOGNON, F.; DALLA CORTE, A. P.; RODRIGUES, A. L.; MAAS, G. C. B. Ajuste de equações hipsométricas para a estimação da altura total de indivíduos jovens de teca. Científica, v. 43, n. 4, p. 400406, 2015. Disponível em: <http://dx.doi.org/10.15361/1984-5529.2015v43n4p400406>. doi: $10.15361 / 1984-5529.2015 v 43 n 4 p 400-406$

SILVA, D. M.; BATALHA, M. A. Defense syndromes against herbivory in a cerrado plant community. Plant Ecology, v. 212, n. 2, p. 181-193, 2011. Disponível em: < http://link.springer.com/article/10.1007\%2Fs11258-010-9813-y>. doi: 10.1007/ s11258-010-9813-y

SILVA, A. M. L.; LOPES, S. F.; VITORIO, L. A. P.; SANTIAGO, R. R.; MATTOS, E. A.; TROVÃO, D. M. B. M. Plant functional groups of species in semiarid ecosystems in Brazil: wood basic density and SLA as an ecological indicator. Brazilian Journal of Botany, v. 37, n. 3, p. 229-237, 2014. Disponível em: < http://link.springer.com/article/10.1007/s40415-014-0063-4>. doi: 10.1007/s40415014-0063-4

SOUZA, B. C.; OLIVEIRA, R. S.; ARAÚJO, F. S.; LIMA, A. L.; RODAL, M. J. N. Divergências funcionais e estratégias de resistência à seca entre espécies decíduas e sempre verdes tropicais. Rodriguésia, v. 66, n. 1, p. 021-032, 2015. Disponível em: <http://rodriguesia.jbrj.gov.br>. doi: 10.1590/2175-7860201566102

VERGUTZ, L.; MANZONI, S.; PORPORATO, A.; NOVAIS, R. F.; JACKSON, R. B. Global resorption efficiencies and concentrations of carbon and nutrients in leaves of terrestrial plants. Ecological Monographs, v. 82, n. 1, p. 205-220, 2012. Disponível em: <https://earth.stanford.edu/jacksonlab/sites/default/files/em2012a.pdf>. doi: $10.1890 / 11-0416.1$

VIANI, R. A. G., RODRIGUES, R. R., DAWSON, T. E., LAMBERS, H., OLIVEIRA, R. S.: Soil $\mathrm{pH}$ accounts for differences in species distribution and leaf nutrient concentrations of Brazilian woodland savannah and seasonally dry forest species, Perspectives in Plant Ecology, Evolution and Systematics, v. 16, n. 2, p. 64-74, 2014. Disponível em: <http://dx.doi.org/10.1016/j.ppees.2014.02.001>. doi:10.1016/j.ppees.2014.02.001. 